\title{
A COSMETOVIGILÂNCIA COMO INSTRUMENTO PARA A GARANTIA DA QUALIDADE NA INDÚSTRIA DE PRODUTOS COSMÉTICOS
}

\author{
BEHRENS, Isabela ${ }^{1}$; CHOCIAI, Jorge Guido²
}

1- Farmacêutica e aluna do curso de especialização "Gestão da Qualidade na Produção de Cosméticos, Medicamentos e Alimentos" - PUC/PR.

2- Professor da Disciplina de Tecnologia de Cosméticos do Curso de Farmácia da UFPR.

\section{RESUMO}

\begin{abstract}
A Agência Nacional de Vigilância Sanitária (ANVISA) vem editando diversas Portarias e Resoluções com o objetivo de regulamentar a produção, a comercialização e a fiscalização do setor de cosméticos no Brasil. No início do ano de 2006 entrou em vigor a resolução RDC 332 de 01 de dezembro de 2005, que tratam da implantação do sistema de Cosmetovigilância em todas as empresas fabricantes e/ou importadoras de Produtos de Higiene Pessoal, Cosméticos e Perfumes. Este sistema foi criado para facilitar a comunicação, por parte do usuário, sobre problemas decorrentes do uso, defeitos de qualidade ou efeitos indesejáveis, e também para melhorar o acesso do consumidor às informações sobre os produtos.

Palavras-chave: cosmetovigilância, fiscalização de produtos cosméticos, qualidade de produtos cosméticos.
\end{abstract}

\begin{abstract}
The Agency of Sanitary Monitoring (Agência Nacional de Vigilância Sanitária - ANVISA) has been editing many laws intending to regularize the production and commercialization of cosmetic products, and in the beginning of the year 2006 a new resolution came up: the RDC 332 of December 1st 2005, which discourse on the implantation of a system of "Cosmetomonitoring" (Cosmetovigilância) in every manufacturing company and/or importer of Personal Hygiene products, cosmetics and perfume in Brazil. This system has been created to facilitate the communication between the client and the company, especially about problems due to the use of a product, quality defects or undesirable effects.
\end{abstract}

Key words: cosmetovigilância; cosmetic products monitoring; quality of cosmetic products.

\section{INTRODUÇÃO}

A Cosmetovigilância é um novo instrumento para a vigilância sanitária implantado no setor de produtos cosméticos, estruturado para suprir as necessidades de maior controle e garantia de qualidade desses produtos, e que, se praticada com o rigor necessário, proporcionará significativos benefícios para a indústria e para o consumidor, a partir da disponibilização de produtos com a qualidade necessária para garantir a sua segurança e eficácia.

\section{DESENVOLVIMENTO}

Cosméticos são preparações constituídas por substâncias naturais ou sintéticas, de uso externo nas diversas partes do corpo humano, pele, sistema capilar, unhas, lábios, etc, com o objetivo exclusivo ou principal de limpá-los, perfumá-los, alterar sua aparência e ou corrigir odores corporais e ou protegê-los ou mantê-los em bom estado (ANVISA, 2005). 
A grande preocupação com a segurança dos produtos cosméticos decorre principalmente do fato desses produtos serem considerados de venda livre, ou seja, o consumidor pode adquiri-los quando desejar, de acordo com suas preferências pessoais, sem a interferência de um profissional da saúde, devendo-se considerar também o crescente consumo mundial desses produtos.

Nos Estados Unidos, a FDA (Food and Drug Administration) é o órgão responsável pelo controle dos produtos cosméticos e que administra apenas a obrigatoriedade de controle após a sua liberação para o mercado. Matérias-primas e produtos acabados não são analisados ou aprovados pela $F D A$. A organização não tem a autoridade para exigir das indústrias de produtos cosméticos, testes de segurança em seus produtos antes de sua comercialização (MEYERS, 2007).

O controle e vigilância sanitária dos produtos cosméticos em alguns países da Europa são realizados de uma forma um pouco diferente.

A França adotou o sistema de Cosmetovigilância (Cosmetovigilance) para garantir a qualidade e segurança dos produtos comercializados no País. A definição de Cosmetovigilância está descrita no artigo L5131-9 da lei 2004-806 de 9 de agosto de 2004 do Código de Saúde Pública da França (Code de la Sanité Publique), e nele consta que todo profissional da saúde que constatar um efeito indesejável causado por um produto cosmético deverá fazer uma declaração ao diretor geral da Agência Francesa de Segurança Sanitária de produtos de saúde. Os fabricantes, os representantes, os responsáveis pela comercialização de produtos importados e os seus distribuidores devem participar do Sistema Nacional de Cosmetovigilância (Système National de Cosmetovigilance) (FRANCE, 2004).

AANVISA é o órgão nacional competente que atua na vigilância sanitária do setor de cosméticos no Brasil. A legislação sanitária do Brasil tem sido atualizada de forma a permitir a fiscalização do setor de produtos cosméticos, dentro do atual estágio de modernidade desses produtos. A legislação tem sido adaptada às exigências do Mercosul, da União Européia e demais países, não só para facilitar os processos de importação e exportação de produtos cosméticos, como também, para garantir maior margem de segurança ao uso desses produtos.

Assim, no início do ano de 2006 foi publicada uma nova resolução, a RDC 332 de 01 de dezembro de 2005, que determina a implantação do sistema de Cosmetovigilância em todas as empresas fabricantes e/ou importadoras de Produtos de Higiene Pessoal, Cosméticos e Perfumes (ANVISA, 2005).

\section{RESOLUÇÃO-RDC Nº. 332, DE $1^{\circ}$. DE DEZEMBRO DE 2005}

A Diretoria Colegiada da Agência Nacional de Vigilância Sanitária, no uso da atribuição que lhe confere o art. 11, inciso IV, do Regulamento da Anvisa, aprovado pelo Decreto no 3.029, de 16 de abril de 1999, c/c o art. 111, inciso I, alínea "b", $\S 1^{\circ}$ do Regimento Interno aprovado pela Portaria $n^{\circ} .593$, de 25 de agosto de 2000, republicada em 22 de dezembro de 2000, em reunião realizada em 28 de novembro de 2005, considerando a missão precípua da Vigilância Sanitária de prevenir agravos à saúde, a ação reguladora de garantia de qualidade de produtos e serviços que inclui a aprovação de normas e suas atualizações, bem como a fiscalização de sua aplicação; considerando que a legislação sanitária vigente se aplica a produtos nacionais e importados, provenientes dos Estados Partes do Mercosul e de outros países (produtos extra-zona); considerando a necessidade de avançar e aprofundar o cumprimento dos padrões de qualidade dos Produtos de Higiene Pessoal, Cosméticos e Perfumes produzidos no país; considerando a responsabilidade dos fabricantes de garantir a segurança e eficácia dos Produtos de Higiene Pessoal, Cosméticos e Perfumes; considerando a necessidade de cumprir-se o s requisitos obrigatórios relacionados à comprovação da segurança e eficácia dos Produtos de Higiene Pessoal, Cosméticos e Perfumes; considerando a importância de compatibilizar os regulamentos nacionais com os instrumentos harmonizados no âmbito do Mercosul, em especial a Resolução GMC nº. 19/05; considerando a Consulta Pública realizada por meio da Portaria GM n. 15, de 5 de janeiro de 2005 (DOU 06/01/05); considerando a importância do assunto, adotou a seguinte Resolução da Diretoria Colegiada, e eu Diretor-Presidente, determino a sua publicação.

Art. $1^{\circ}$ As empresas fabricantes e/ou importadoras de Produtos de Higiene Pessoal Cosméticos e Perfumes, instaladas no território nacional deverão implementar um Sistema de Cosmetovigilância, a partir de 31 de dezembro de 2005.

Parágrafo único. O Sistema de Cosmetovigilância de Produtos de Higiene Pessoal, Cosméticos e Perfumes, facilitará a comunicação, por parte do usuário, sobre problemas decorrentes do uso, defeitos de qualidade ou efeitos indesejáveis e o acesso do consumidor à informação. 
Art. $2^{\circ}$ As empresas fabricantes e/ou importadoras de Produtos de Higiene Pessoal Cosméticos e Perfumes, instaladas no território nacional deverão manter registro dos relatos de cosmetovigilância, e avaliá-los.

Art. 3 Se do resultado da avaliação dos relatos identificarem situações que impliquem em risco para a saúde do usuário, as empresas fabricantes e/ou importadoras dos Produtos de Higiene Pessoal, Cosméticos e Perfumes instaladas no território nacional deverão notificar à Autoridade Sanitária Federal do Brasil (Anvisa) e dos Estados Partes do Mercosul envolvidos (ANVISA, 2005).

Esta resolução da ANVISA foi elaborada a partir da resolução do Mercosul MERCOSUR/GMC/RES. No 19/05, a qual determina aos países integrantes do Mercosul a implantação de um sistema de Cosmetovigilância para garantir a segurança e eficácia dos produtos cosméticos, função esta delegada aos organismos nacionais competentes de cada país associado (MERCOSUL, 2005).

O sistema de Cosmetovigilância foi criado para facilitar a comunicação, sobre problemas decorrentes do uso, defeitos de qualidade ou efeitos indesejáveis, e também para melhorar o acesso do consumidor às informações sobre o produto. Através do sistema de cosmetovigilância, as empresas fabricantes e/ou importadoras de produtos cosméticos deverão manter registro de todos os relatos feitos pelos consumidores em relação a problemas causados por cosméticos, e avaliá-los. A partir dessa avaliação devem ser tomadas medidas com o intuito de garantir a qualidade e segurança do produto em questão. No caso de situações que impliquem em risco à saúde do usuário, as empresas deverão notificar a ANVISA sobre o relato (ANVISA, 2005).

Problemas relacionados ao uso de produtos cosméticos, desde fatos relacionados à instabilidade dos produtos e defeitos de embalagens, até o desencadeamento de irritações, reações alérgicas e infecções, sempre foram constatadas, porém, nunca foram devidamente registrados e nem encaminhadas a ANVISA, a qual não tinha controle das ocorrências, da sua freqüência e nem das substâncias a elas relacionadas. Assim, na maioria das vezes, o problema não tinha o encaminhamento necessário, resultando no simples abandono do uso do produto pelo consumidor. A cosmetovigilância é uma tentativa de vencer esta barreira na atuação da vigilância sanitária em produtos cosméticos.

A cosmetovigilância relaciona-se diretamente à edição da Portaria $n^{\circ} 348$ de agosto de 1997, que determina a todos os estabelecimentos produtores de Produtos de Higiene Pessoal, Cosméticos e Perfumes, o cumprimento das Diretrizes estabelecidas no Regulamento Técnico - Manual de Boas Práticas de Fabricação para Produtos de Higiene Pessoal, Cosméticos e Perfumes. (ANVISA) e, segundo Trevisan (2006), pode ser entendida também como uma forma do consumidor avaliar se o fabricante cumpre efetivamente as recomendações das BPF's, pois se elas forem cumpridas, o número de ocorrências de não-conformidades e seus efeitos indesejáveis será reduzido ou talvez inexistente (TREVISAN, 2006).

Um exemplo simples de uma possível correlação entre o não-cumprimento das BPF's e suas conseqüências é a ausência de Procedimentos Operacionais Padrões (POP's), que pode gerar produtos com características diferentes lote a lote, que levam à insatisfação do cliente.

A Cosmetovigilância tem um paralelo com a atribuição da Farmacovigilância, esta, relacionada à detecção, avaliação, compreensão e prevenção dos efeitos adversos ou quaisquer problemas relacionados a medicamentos. Tem, portanto, a função de estudar, compilar os dados e propor alterações quanto aos medicamentos que eventualmente causem reações adversas aos consumidores (IDEC, 2007). A analogia, em princípio, demonstrase coerente até porque permite ao consumidor o direcionamento de sua reclamação, dúvida ou denúncia a um canal especializado e com poder de decisão para deflagrar mudanças na produção, distribuição, armazenamento, comercialização e publicidade de produtos cosméticos (IDEC, 2007).

Complementando a implantação da Cosmetovigilância, a ANVISA implantou o NOTIVISA, que é um sistema informatizado para receber notificações de eventos adversos e queixas técnicas relacionados aos produtos sob vigilância sanitária, incluindo os cosméticos. Através do NOTIVISA, podem cadastrar-se profissionais de saúde ou instituições/entidades. Os cidadãos poderão notificar eventos adversos ou queixas técnicas através de formulário de notificação. Poderão ser notificados casos confirmados ou suspeitos de eventos adversos ou queixas técnicas sobre produtos. Ao receber a notificação, os órgãos integrantes do SNVS analisarão a notificação de acordo com a gravidade e risco do evento adverso ou da queixa técnica. Terão acesso às informações o notificador, as vigilâncias sanitárias dos Municípios e dos Estados e a ANVISA. Os dados referentes às notificações servirão para subsidiar o Sistema Nacional de Vigilância Sanitária (SNVS) para identificar reações adversas ou efeitos não-desejados dos produtos, aperfeiçoarem o conhecimento dos efeitos dos produtos e, quando indicado, alterar recomendações sobre seu uso e cuidados e regular os produtos comercializados no País e, de forma geral, promover ações de proteção à Saúde Pública (ANVISA, 2007). 


\section{CONSIDERAÇÕES FINAIS}

O sistema de Cosmetovigilância foi implantado para garantir a qualidade final dos produtos cosméticos, tendo em vista, principalmente a segurança, eficácia, e informação à ANVISA, ao fabricante e ao consumidor. Esse sistema poderá proporcionar inúmeros benefícios ao setor de cosméticos como um todo.

Os benefícios serão decorrentes da implantação de um canal específico para o registro dos eventos adversos e queixas técnicas sobre produtos de higiene pessoal, cosméticos e perfumes, e principalmente pelo banco de dados que poderá ser gerado a partir de tal procedimento. Essas informações, anteriormente, se perdiam pela inexistência de um sistema próprio para o seu registro.

Contudo, esse novo instrumento precisa alcançar e ser incorporado pelo setor industrial, pelos profissionais de saúde e também pelo consumidor.

Do total de 5064 notificações sobre todos os produtos sujeitos à vigilância sanitária, efetuados no período de dezembro de 2006 a agosto de 2007, 16 notificações referem-se a eventos adversos ou queixas técnicas decorrentes de produtos cosméticos (ANVISA, 2007).

O curto período de tempo de implantação do sistema de cosmetovigilância e o número de notificações efetuadas, ainda não são significativos para permitir uma avaliação segura do quadro geral a respeito da qualidade dos produtos de higiene pessoal, cosméticos e perfumes no Brasil.

\section{REFERÊNCIAS}

ANVISA.

AGÊNCIANACIONAL DE VIGILÂNCIA SANITÁRIA. Guia de estabilidade de produtos cosméticos. 1. ed. Brasília: ANVISA, 2004.

ANVISA.

AGÊNCIA NACIONAL DE VIGILÂNCIA SANITÁRIA. Guia para Avaliação de Segurança de Produtos Cosméticos. 1. ed. Brasília: ANVISA, 2003.

ANVISA. Notivisa - Sistema de Notificação em Vigilância Sanitária. Ministério da Saúde. Disponível em:

<www.anvisa.gov.br/hotsite/notivisa/index.htm>. Acesso em 10 set. 2007.

BEZERRA, S. V.; REBELLO, T. Guia de produtos cosméticos. 4. ed. São Paulo: Editora Senac, 2003. 104 p.

BRASIL. Ministério da Saúde. Agência Nacional de Vigilância Sanitária. Portaria $\mathrm{n}^{\circ} 13$ de 05 de Janeiro de 2005. Torna pública a proposta de Projeto de Resolução de Auto-Inspeções de Boas Práticas de Fabricação e Controle na Área de Produtos de Higiene Pessoal, Cosméticos e Perfumes e dá outras providências. Disponível em < http://www. anvisa. gov.br/e-legis> Acesso em: 06 fev. 2007.

BRASIL. Ministério da Saúde. Agência Nacional de Vigilância Sanitária. Portaria n 348 de 18 de Agosto de 1997. Determina a todos estabelecimentos produtores de Produtos de Higiene Pessoal, Cosméticos e Perfumes, o cumprimento das Diretrizes establecidas no Regulamento Técnico - Manual de Boas Práticas de Fabricação para Produtos de Higiene Pessoal, Cosméticos e Perfumes. Disponível em < http://www. anvisa. gov.br/e-legis>. Acesso em: 10 dez. 2006.

BRASIL. Ministério da Saúde. Agência Nacional de Vigilância Sanitária. Resolução RDC n²11 de 14 de julho de 2005. Ficam estabelecidas a Definição e a Classificação de Produtos de Higiene Pessoal, cosméticos e Perfumes, conforme Anexos I e II desta Resolução. Disponível em: <http://www. anvisa. gov.br/e-legis>. Acesso em: 10 dez. 2006.

BRASIL. Ministério da Saúde. Agência Nacional de Vigilância Sanitária. Resolução RDC n 332 de 01 de dezembro de 2005. As empresas fabricantes e/ou importadoras de Produtos de Higiene Pessoal Cosméticos e Perfumes, instaladas no território nacional deverão implementar um Sistema de Cosmetovigilância, a partir de 31 de dezembro de 2005. Disponível em: <http://www. anvisa. gov.br/e-legis>. Acesso em: 08 nov. 2006.

CHARLET, E. Cosmética para Farmacêuticos. Zaragoza: Editorial Acribia S.A., 1996. p. 17-24.

FRANCE. Agence Française de Securité Sanitaire des Produits de Sanité. Code de La Sanité Publique. Article L5131-9 Loi n 2004-806 du 9 août 2004. Disponível em:

<http://www.legifrance.gouv.fr/WAspad/RechercheSimpleArticleCode?code=CSANPUNL.rcv\&art=L5131-9\&indice=0>. Acesso em 31 jan. 2007. 
IDEC. Qualidade de Produtos e Serviços. Instituto Brasileiro de Defesa do Consumidor. Disponível em:

<http://www.idec.org.br/emacao.asp?id=844\&categoria=12>. Acesso em 05 fev 2007.

MERCOSUL. MERCOSUR/GMC/RES. No 19/05. Programa de cosmetovigilância em el area de productos de higiene personal, cosméticos e y perfumes. Disponível em: <www.toxiclin.com.br/DOCUMENTOS/MERCOSUR-GMC-RES.htm>. Acesso em 19 dez. 2006.

MEYERS, B. FDA Authority Over Cosmetics. FDA. Disponível em: <http://www.cfsan.fda.gov/ dms/cos-206.html>. Acesso em 22 jan. 2007.

QUIROGA, M; GUILLOT, C. F. Cosmética Dermatológica Práctica. 4. ed. Buenos Aires: El Ateneo, 1973. p.18-40.

REINHOLD, E. Consumer Advice and Publications on Food Safety, Nutrition, and Cosmetics. FDA. Disponível em: <http://www.cfsan.fda.gov/ Ird/advice.html\#cosmetics>. Acesso em: 25 jan. 2007.

SANTOS, I. M. N. S. R. Workshop Segurança e Avaliação por Similaridade e Cosméticos. ANVISA. Disponível em: <www.anvisa.gov.br/divulga/eventos/workshop_cosmeticos/idalina.ppt>. Acesso em 02 mar. 2007.

TOXICLIN. Cosmetovigilância. Toxiclin Serviços Médicos LTDA. Disponível em:

<http://www.toxiclin.com.br/cosmetovig.htm>. Acesso em 15 dez. 2006.

TREVISAN, C. A. Cosmetovigilância e BPF. Cosmetics e Toiletries, São Paulo, v. 18, p.44, nov/dez. 2006.

VIGLIOGLIA, P. A.; RUBIN, J. Cosmiatria II . 2.ed. Buenos Aires: Americana de Publicaciones AS, 1991. p. 22-34. 